\title{
Un análisis del vínculo abuelos nietos-adolescentes reflexión sobre la transmisión generacional
}

\author{
Alejandro León Klein \\ Universidad de Guanajuato, México \\ Lorena Isis Hernández Basilio \\ Universidad Nacional Autónoma de México
}

María Cristina Rodríguez García

Universidad De Guanajuato, México

Un análisis del vínculo abuelos nietos-adolescentes reflexión sobre la transmisión generacional Resumen: El presente trabajo se basa en una investigación exploratoria y descriptiva sobre las relaciones intergeneracionales abuelosnietos adolescentes, buscando comprender, desde la perspectiva del adolescente, las comunicaciones, significaciones y afectos que se gestan en torno a su vínculo transgeneracional, diferenciando las relaciones que establecen con los abuelos y las abuelas, así como posibles diferencias vinculares entre los abuelos paternos y los abuelos maternos. Existen diferencias basadas en la selección voluntaria de actividades con los abuelos de la línea materna, mientras que con los abuelos de la línea paterna parecen reflejar actividades de índole obligatoria que no forman parte de un agrado aparente, pero sí forman parte de vínculos integrativos de su identidad y su vínculos tanto intergeneracionales cómo transgeneracionales.

Palabras clave: Abuelos. Nietos adolescentes. Generaciones. Selectividad.

Análise da relação entre avós e netos-adolescentes: reflexão sobre a transmissão intergeracional Resumo: Este artigo é baseado em uma pesquisa descritiva e de campo sobre relações intergeracionais entre netos adolescentes e seus avós, procurando compreender, a partir da perspectiva dos adolescentes, comunicações, significados e emoções que estão se desenvolvendo em torno da sua ligação transgeracional, diferenciando as relações de avós com avós, bem como possíveis diferenças relacionais entre avós paternos e os avós maternos. Entre as conclusões, destaca que a ligação entre as gerações implica termos simbólicos e reais dentro de uma família. A seletividade, o poder de escolher com qual avô executar tarefas e atividades, apareceu como um fator chave para entender as características das relações transgeracionais e intergeracionais.

Palavras-chave: Avós. Netos adolescentes. Gerações. Seletividade.

\begin{abstract}
An Analysis of the Ties between Grandparents and their Adolescent Grandchildren: a reflection on generational transmission

Abstract: This study is based on an exploratory and descriptive study about the intergenerational relations between grandparents and their adolescent grandchildren. It is an effort to understand, from the perspective of the adolescent, the communications, meanings and affections associated to these transgenerational ties. It seeks to identify differences in relations with grandfathers and grandmothers, as well as possible differences in ties between paternal and maternal grandparents. Differences were identified based on a voluntary choice to engage in activities with maternal grandparents. Meanwhile, relations with paternal grandparents seem to reflect activities of an obligatory nature, which are not related to apparent pleasure, but to be an integral part of the adolescents' identities and their intergenerational and transgenerational ties.
\end{abstract}

Keywords: Grandparents. Adolescent grandchildren. Generations. Selectivity. 


\section{Introducción}

La población en México sufrió importantes transformaciones a lo largo del siglo 20. Una de las más importantes ha sido la llamada transición demográfica producto del descenso de la mortalidad a partir de los años treinta y el de la fecundidad en los años setenta. Como resultado de estos cambios, la población se ha incrementado, la estructura por edad de la población se ha modificado y la cantidad relativa de adultos mayores ha aumentado, esto es, en México, la población está envejeciendo (CONAPO, 2013). Puesto en cifras, en 2010 residían en México poco más de diez millones de adultos mayores (INEGI, 2011; CONAPO, 2013). Entre 1990 y 2010 su número pasó de 5 a 10 millones, presentándose un incremento porcentual, respecto al total de la población, de 2.8; esto es, pasaron del 6.2 al 9 por ciento de la población total (INEGI, 2011). La esperanza promedio de vida al nacer en México pasó de 36 años en 1950 a 74 años en el año 2000. De acuerdo con proyecciones de CONAPO (2013), para 2020 la población de adultos mayores alcanzará su tasa máxima de crecimiento (4.2\%) con 14 millones de individuos; $12.1 \%$ de la población. A partir de ese año, el ritmo de crecimiento demográfico comenzaría a disminuir, hasta alcanzar un crecimiento negativo (-1.58\%) en 2050, cuando se prevé que habrá cerca de 34 millones de adultos mayores que representarán el $27.7 \%$ de la población total (VILLAGÓMEZ ORNELAS, 2009). Por otro lado, las complejas y rápidas transformaciones políticas, económicas y sociales coinciden en cambios significativos en la vida familiar y sus vínculos (RIZZINI, 2001). Cada vez más los adolescentes viven y crecen dentro de estas nuevas configuraciones familiares (WAINERMAN, 1996). Bengtson (2001) sugiere así que los abuelos desempeñan un papel cada vez más importante en las familias. Los efectos combinados de la mayor esperanza de vida (lo que representa un curso de vida más largo junto a los nietos) y la fertilidad descendente (pocos nietos) pueden tener el efecto secundario de un mayor relacionamiento abuelos-nietos, además de abuelos que compiten por la atención de sus pocos nietos (UHLENBERG, 2005). Moragas (1997) destaca igualmente que la mayor longevidad propicia una coexistencia más larga entre los abuelos y sus nietos. Harper (2003) indica que el incremento de la longevidad se puede relacionar al surgimiento de roles de mayor acercamiento entre aquéllos. De esta manera, el rol de las personas de edad se modifica, pasando de ser una persona pasiva que necesita cuidados y protección, a ser un miembro activo de la familia, concediendo protección y cuidados.

Estos cambios sociales y familiares implican un cambio profundo en los papeles del abuelo y de la abuela (WILCOXON, 1987). El hecho es que cada vez más los adolescentes son criados por sus abuelos (EHRLE; DAY, 1994). Para el año 2005 se estimaba que había 4.5 millones de niños viviendo con sus abuelas en Estados Unidos, lo que representa un incremento del $30 \%$ tomando como parámetro la década 1990-2000 (AARP, 2005). Otros datos aumentan este número a 5.8 millones de niños y adolescentes para el año 2002 (U.S. CENSUS BUREAU, 2002).

Hay varias razones por las que los abuelos toman plena responsabilidad por sus nietos. Algunas de estas razones son: abuso de drogas, embarazo adolescente, divorcio, padres que viven solos, padres en régimen de prisión, abuso infantil, violencia doméstica, dolencia mental y física y descuido (LEVER; WILSON, 2005). De una u otra manera, cuando los abuelos se hacen responsables del bienestar de sus nietos esto tiende a modificar la estructura familiar (KLEIN, 2009; 2010). Diversas investigaciones señalan que surgen nuevas tendencias de lo que es considerado familia y los roles que se esperan de los llamados "familiares" (WIDMER, 2004). De esta manera se hace necesario revisar qué se considera familiares significativos desde las nuevas configuraciones familiares (stepfamilies) ampliando el espectro de estudio más allá de la relación matrimonial o filial (GANONG; COLEMAN, 2004). Contrariamente a la hipótesis del aislamiento de la familia nuclear, diversas investigaciones indican que los diversos parientes mantienen relaciones emocionales, de apego y mantenimiento de contactos regulares, experimentando diversas formas de soporte mutuo (ADAMS, 1999). Nos interesa especialmente, en lo que hace a esta investigación, el concepto del Beanpole como estructura familiar cuyos miembros provienen de varias generaciones, pero con pocos miembros en cada generación (BENGSTON; ROSENTHAL; BURTON, 1990). Estas configuraciones demuestran fuertes conexiones intergeneracionales (COLEMAN, 1988) que comúnmente incluyen abuelos, tíos y tías. Por tanto desde la perspectiva de jóvenes y adolescentes, éstos reciben cuidado y atención de un gran número de miembros de familias interconectadas, que incluyen generaciones previas (FURSTENBERG; HUGHES, 1995). La importancia del papel de estos vínculos en los procesos de transmisión generacional conduce a un análisis que implica reconocer la importancia de los antecesores, procesar vínculos, resignificar tradiciones de forma generacional (KLEIN, 2013).

\section{Metodología}

Metodológicamente, en este primer abordaje estamos especialmente interesados en conocer la opinión y la percepción de los propios nietos y nietas, sobre las relaciones y los vínculos que establecen o 
no con sus respectivos abuelos y abuelas; incluyendo la diferencia entre abuelos paternos y abuelos maternos. Se exploran, en particular, los lazos, relaciones y roles que se construyen con los abuelos. Las características de los 177 adolescentes estudiados fueron: jóvenes adolescentes de entre 16 y 19 años de edad, escolarizados, de nivel medio superior, seleccionados a través de un muestreo probabilístico, que estuvieran bajo la condición de tener abuelos maternos y paternos vivos. Por lo que los resultados exploratorios que se generaron partieron básicamente de las percepciones de los adolescentes y de las características de las relaciones con las y los abuelos.

Para la realización de este estudio se retomó y modificó el cuestionario usado por la Universidad de Oxford para el Instituto de Envejecimiento, liderado por el Dr. George Leeson. Las modificaciones realizadas se plantearon con el objetivo de integrar preguntas relacionadas con la realidad local mexicana. La información que de este cuestionario se obtuvo fue dividida de la forma siguiente:

Sección A: datos generales de la relación de los adolescentes con los y las abuelas-nietos;

Sección B: datos sobre las actividades que aportan información sobre la percepción y significancia emocional de relaciones que los adolescentes establecen con los abuelos a según línea filial y género, contestada a través de la escala Likert, de la que se obtuvieron datos específicos sobre: frecuencia y tipo de actividades que se realizan con los abuelos y abuelas; participación activa del adolescente con respecto al género abuelo y abuela; participación activa del abuelo/a hacia el adolescente desde la perspectiva del adolescente; significancia emocional que da el adolescente al vínculo intergeneracional entre él y sus abuelos tanto de línea materna, como de línea paterna.

\section{Pregunta y objetivos de investigación}

El objetivo es identificar las características de la relación entre abuelos y nietos desde la perspectiva del adolescente. Los objetivos específicos son: conocer las características que perciben los adolescentes en la relación abuelos-nietos; comparar las características entre abuelos paternos y maternos para observar diferencias asociadas a la variable de sexo; y conocer las diferencias que los nietos adolescentes marcan con respecto a sus abuelos y abuelas, tanto línea materna como paterna asociada a la variable vincular-generacional.

Las preguntas de investigación son: ¿cuáles son las características que perciben los adolescentes en la relación con sus abuelos? Con respeto a estas características, ¿existe diferencia en la relación que reportan los adolescentes con el abuelo y/o la abuela? Con respecto a estar características, ¿existe diferencia para el adolescente en su relación entre los abuelos paternos y maternos?

\section{Tabla 1 - Distribución de los grupos de nietos y nietas adolescentes}

\section{Grupo de nietas adolescentes}

Constituido por 83 mujeres, cuyas edades pertenecen a un rango de entre 16 y 17 años, estudiantes de preparatoria, de nivel socioeconómico medio, pertenecientes a familias en las que se reportó que viven en matrimonio.

\section{Grupo de nietos adolescentes}

Constituido por 90 hombres pertenecen a un rango de entre 16 y 17 años, estudiantes de preparatoria, de nivel socioeconómico medio, pertenecientes a familias en las que se reportó que viven en matrimonio.

Los adolescentes en general reportaron tener una mejor relación con su abuela materna, reflejado esta opinión en un $66 \%$ las nietas y un 49\% de los nietos. Marcando una diferencia para su relación con los abuelos hombres en la que los nietos hombres son los que están en mayor contacto con sus abuelos ( 27\%), mientras que las nietas se contactan con ellos en un $12 \%$, como se observa en la figura 1:

La forma de contacto más frecuente (Figura 2), para todo el grupo nietos y nietas, fue en persona 69\%, seguida de llamadas telefónicas en $20 \%$. Las abuelas materna y paterna tienen un porcentaje similar de contacto en persona, 67 y el 69\% respectivamente. Sin embargo, en los abuelos que hay una diferencia significativa teniendo el abuelo materno un porcentaje mayor con un $82.9 \%$ que el abuelo paterno un porcentaje de $46.8 \%$. Es importante señalar que con respecto al abuelo paterno un 50\% no respondió como era la forma de contacto con él. Llama la atención este alto porcentaje de falta de respuestas. 


\section{Figura 1- Mejor relación con los abuelos}

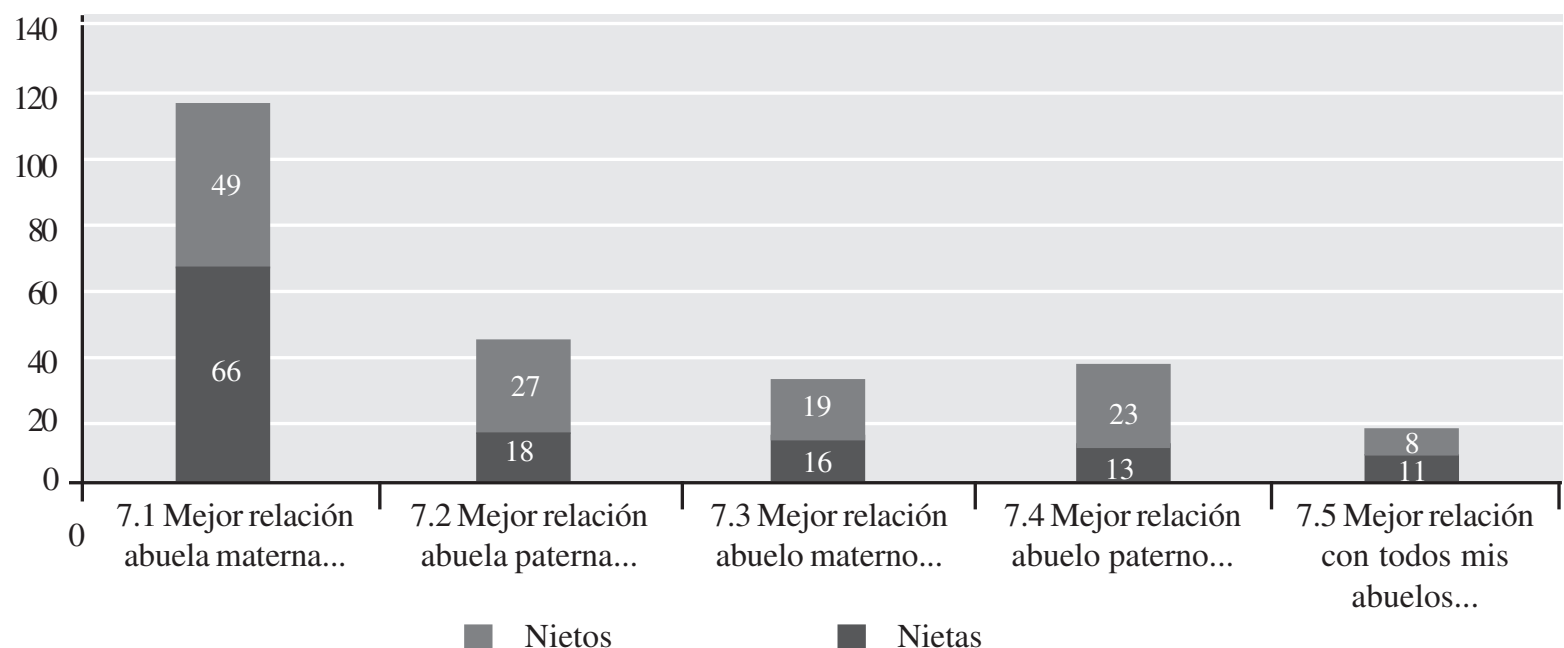

Fuente: Hernández; Rodriguez(2015).

\section{Figura 2 - Contacto y relación con abuelos}

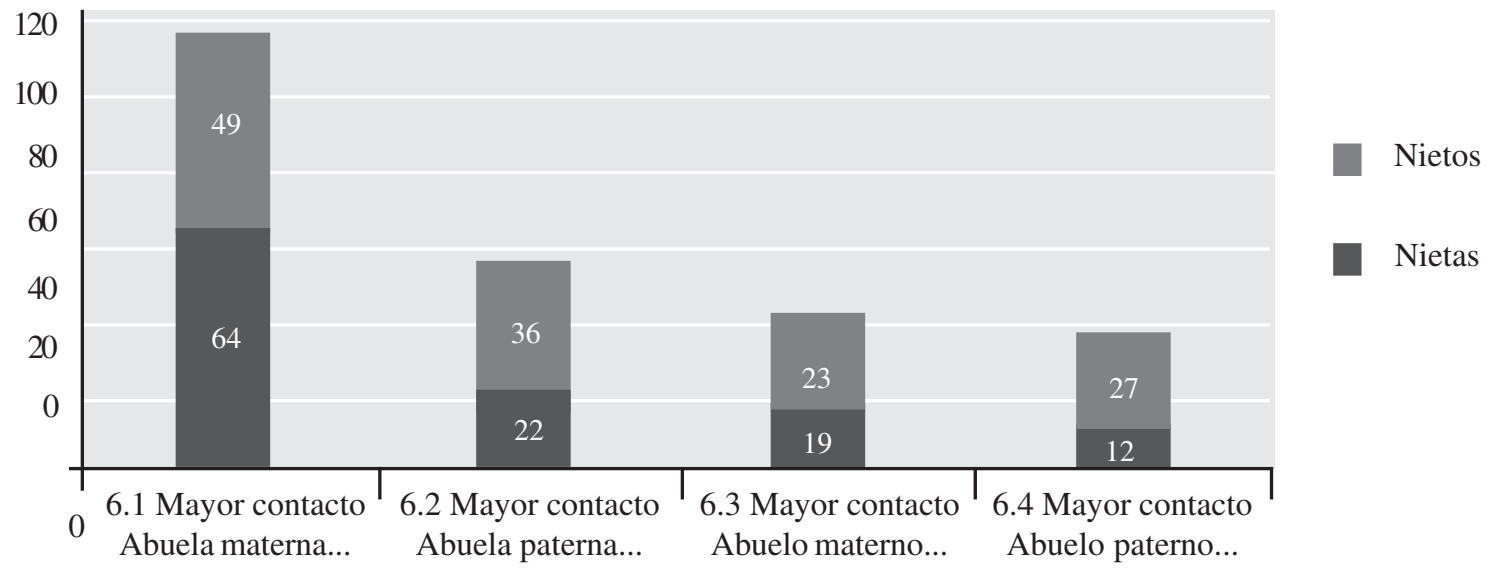

Fuente: Hernández; Rodriguez (2015).

Casi el 50\% de los nietos consideran que la relación que tienen con sus abuelos y abuelas está muy influida por sus padres. Tanto los nietos como las nietas refirieron que la abuela materna es quien los conoce más en un 34\%, mientras que la abuela paterna los conoce en un $13 \%$ de los casos. El abuelo materno es percibido con distancia, sienten que él no los conoce y a su abuelo paterno menos aún (44\%,).

Nuevamente se observa que un $48 \%$ de adolescentes decidió no responder sobre la cercanía con su abuelo paterno. Las nietas manifestaron mayor conocimiento sobre sus abuelas, sin embargo los nietos se mantienen en un porcentaje similar con respecto a los abuelos y las abuelas siendo la respuesta "Más o menos" la más frecuente. Todos los adolescentes refieren ser felices en la relación con sus abuelos. En primer lugar con un $76 \%$ para la materna; segundo lugar con un $72 \%$ para el abuelo materno; en tercer lugar con un $63 \%$ para la paterna y como en los demás casos $28 \%$ para el paterno. 


\section{Resultados sobre la percepción y significancia emocional de relaciones que los adolescentes establecen con los abuelos según línea filial y género}

Sobre el tipo de actividades que realizan con sus abuelos se encontró que los nietos, con ambas abuelas, realizan actividades como conversar (27\%), ver la televisión (19\%) y visitarlas (18\%). En las nietas se observa que las actividades más frecuentes son conversar (37\%), ver televisión (34\%), además en forma muy preferente leen y están en la computadora (16\%) con la materna y en forma casi nula con la abuela paterna. Los nietos salen de casa haciendo visitas familiares y hablan con ambos abuelos, pero en particular se observa una preferencia por el abuelo paterno para vacacionar, ver televisión y jugar juegos de mesa, en porcentajes siete veces más alto que con los abuelos maternos, lo cual podría indicar una predominancia vincular por la línea paterna.

\section{Figura 3 - Actividades significativas de los nietos con sus abuelas}

Nietos y abuelas materna y paterna.

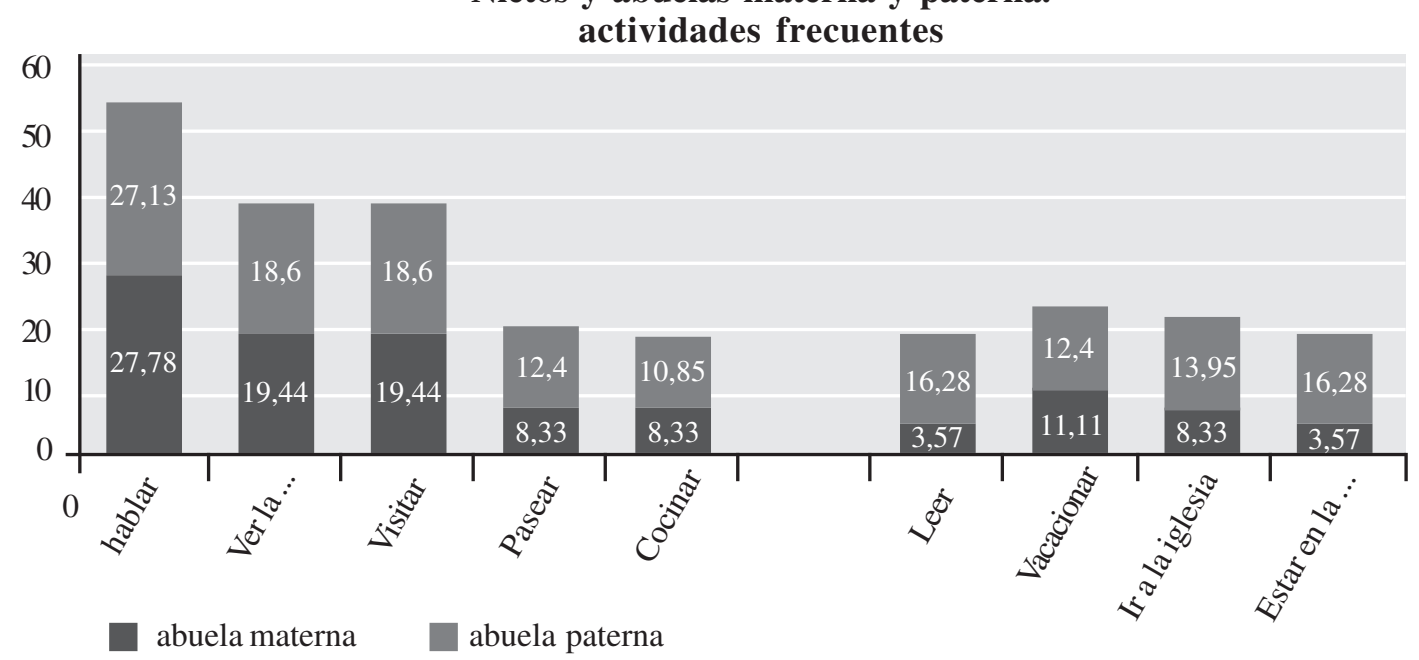

Fuente: Hernández; Rodriguez(2015).

Figura 4 - Actividades significativas de las nietas con sus abuelas

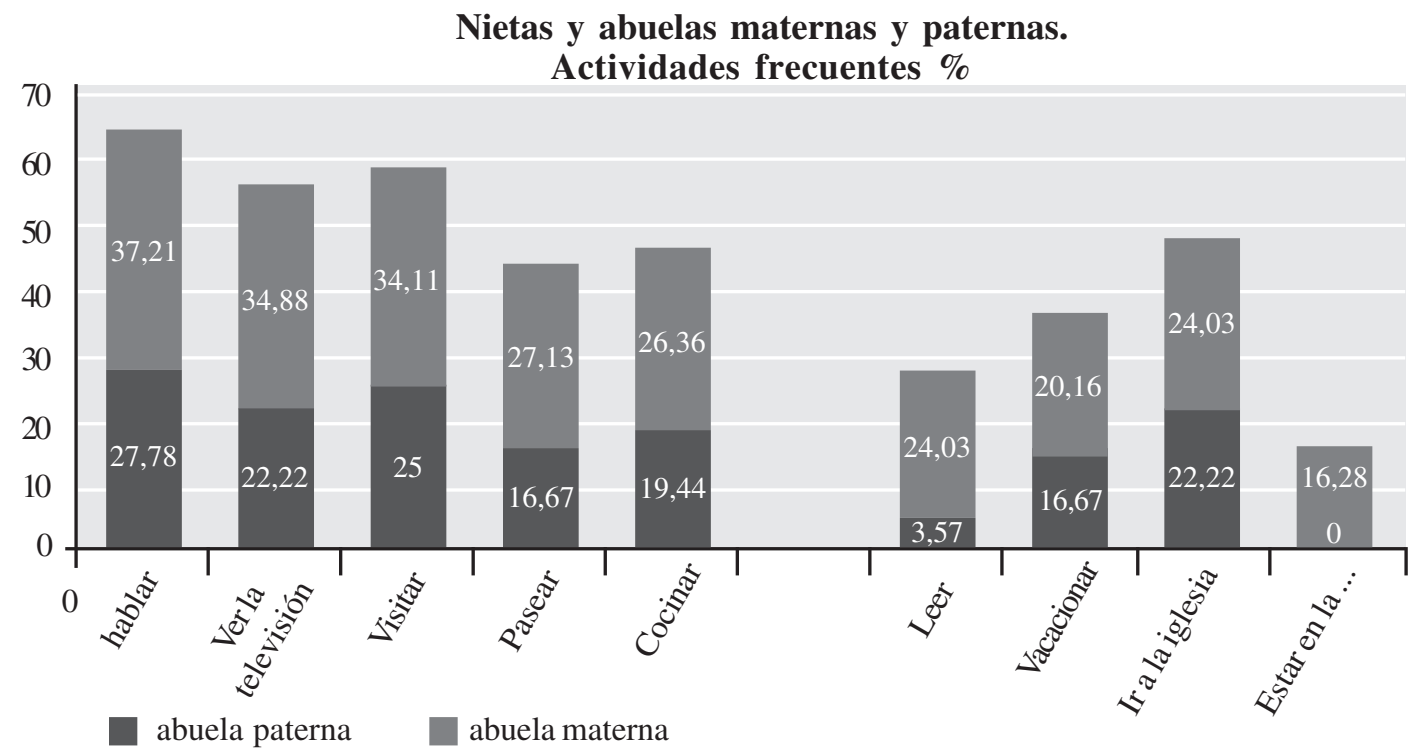

Fuente: Hernández; Rodriguez(2015). 
Sobre la significación emocional, la abuela materna cuenta más sobre sí misma y su historia de vida, la cual puede ser indicador de mayor cercanía afectiva e incluso mayor lealtad mutua (40\% para nietas, 35\% para nietos). Los abuelos paternos transmiten a los nietos la historia familia, las experiencias de juventud y consejos (14\% para nietos, $13 \%$ para nietas), lo cual sucede también con respecto a los abuelos maternos en un porcentaje similar.

Figura 5 - Actividades significativas de las abuelas con sus nietos

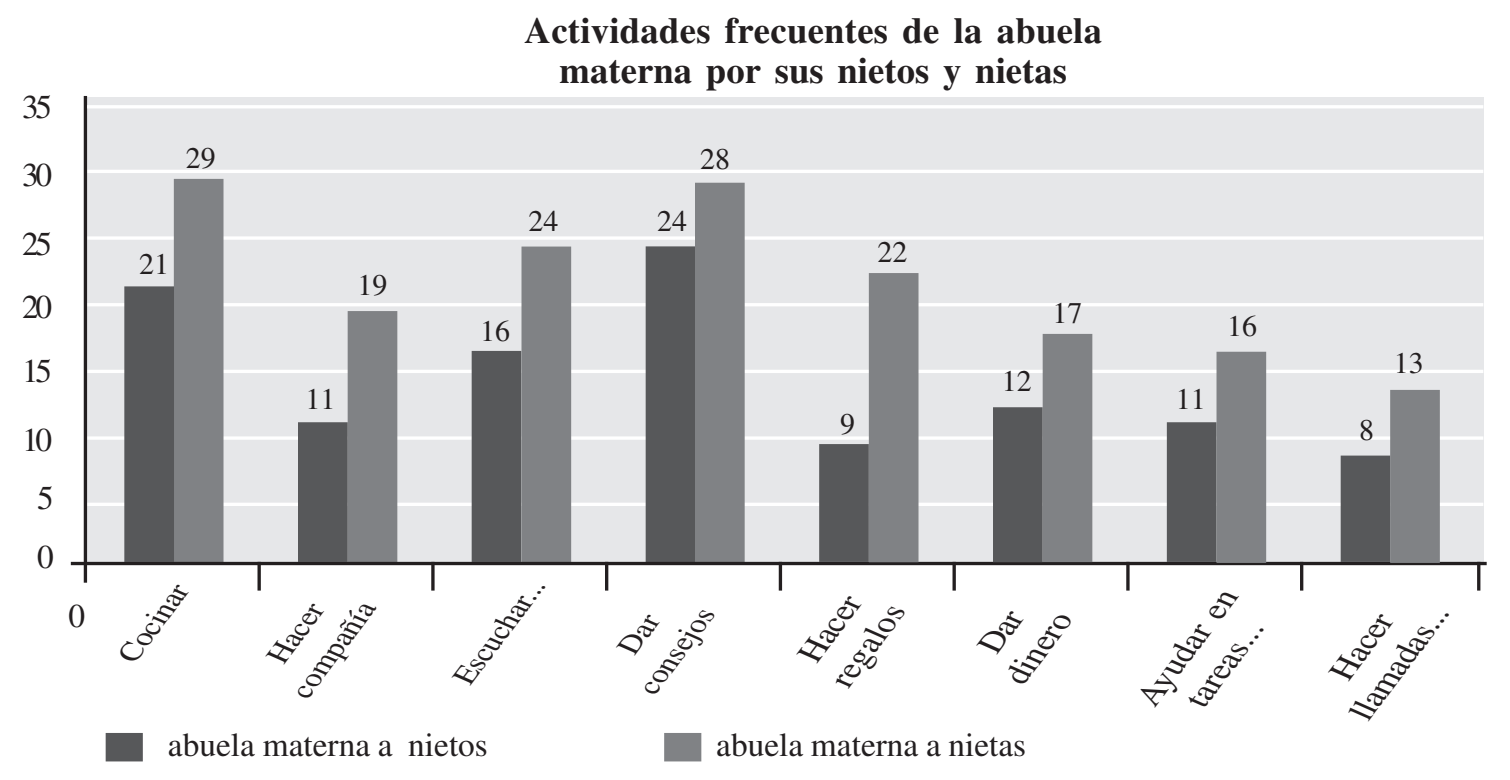

Fuente: Hernández; Rodriguez(2015).

Figura 6 - Actividades significativas de las abuelas paternas con sus nietos.

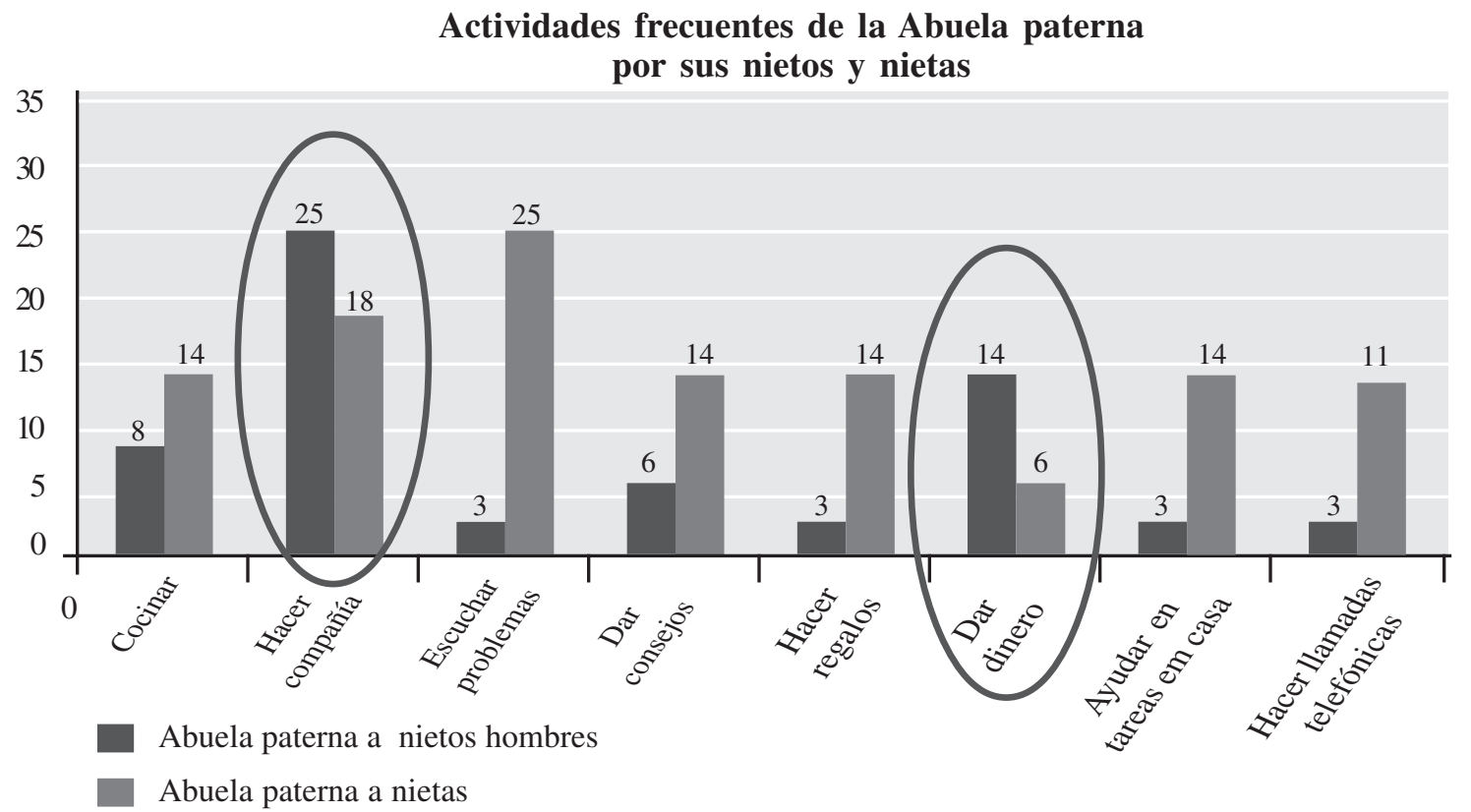

Fuente: Hernández; Rodriguez(2015). 
Figura 7 - Actividades significativas de los abuelos paterno y materno con sus nietos.

Lo que hace el abuelo paterno por sus nietas y nietos

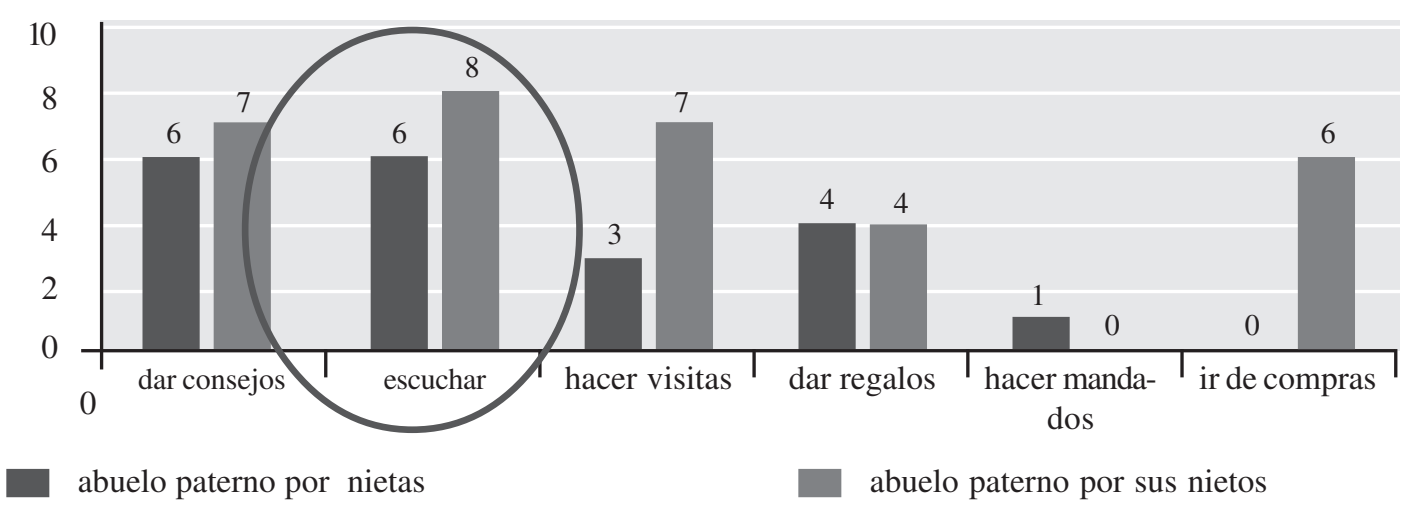

Lo que hace el abuelo materno por sus nietas y nietos

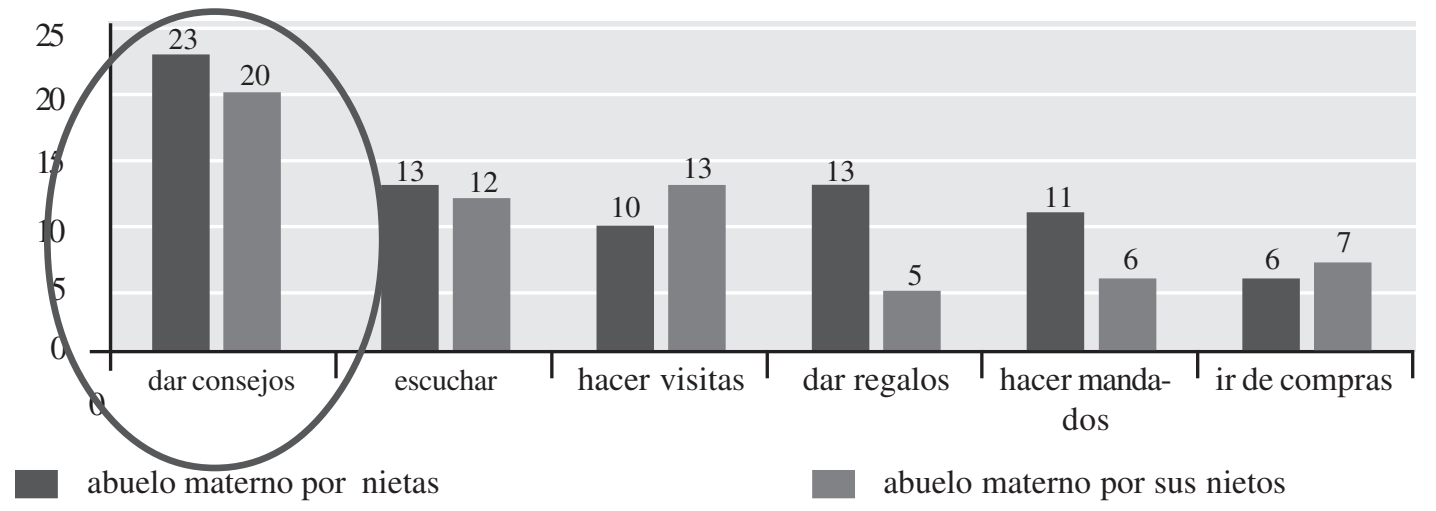

Fuente: Hernández; Rodriguez(2015).

\section{Discusión}

Los resultados obtenidos concuerdan con diversas investigaciones previas donde se señala la relevancia del vínculo abuelos-nietos adolescentes, así como las diferencias existentes en el vínculo entre abuelos y abuelas, además de la predominancia de la línea materna en el vínculo con los abuelos. Se pude destacar que hay actividades que reflejan mandatos sociales que en general se espera en la relación de los nietos (as) con sus abuelos (as), los cuales resultan tareas obligatorias para los y las nietos adolescentes, como son especialmente las visitas regulares a aquéllos.

Un análisis de mayor profundidad nos ha revelado que estas actividades obligatorias no constituyen el contenido ni la forma de transmisión del vínculo generacional, sino que el mismo se desprende desde una selectividad afectiva que realizan los y las nietos y los y las abuelas. El y la adolescente eligen lo que le es significativo y lo que no, es decir, que aunque pueden estar obligado a realizar actividades impuestas por la familia, igualmente seleccionan otras con inmensa carga afectiva y significancia emocional. Por esta razón las actividades selectivas de cooperación son tan significativas en los resultados. En este tipo de vínculo - ver televisión o mirar la computadora - no hay imposición, sino que hay aceptación, cooperación y acompañamiento. Tampoco hay fines educativos (que se depositan en los padres), sino más bien fines afectivos que tiene que ver con un estar presentes, y disfrutar el momento.

Los resultados indican que hay variaciones en cuanto a lo que se espera de los abuelos y las abuelas y que se asocia con características del vínculo masculino o femenino respectivamente el cual constituye una de las formas de vínculo generacional. Es de gran relevancia comprender que constituye un error el asumir que esta transmisión se da generalizadamente hacia un grupo denominado nietos o abuelos, pues existen diferenci- 
as significativas en el vínculo entre nietas y nietas y entre abuelas y abuelas. Es decir: la transmisión generacional se actualiza dependiendo del género o de la línea filial materna o paterna.

\section{Conclusiones}

En general los adolescentes encuestados sienten que la abuela materna mantiene un lugar privilegiado emocionalmente, que los conoce y de la que reciben consejos. Por el contrario afirman que los abuelos no los conocen lo suficiente; no obstante se sienten felices con la relación que mantienen con ambos. A todos los abuelos, tanto paternos como maternos, los contactan por medio de visitas personales y en forma especial hacen llamadas telefónicas a ambas abuelas, pero no a los abuelos. La abuela materna es por la que existe una marcada preferencia tanto para actividades cotidianas, como actividades con carga afectiva y actividades que involucran aspectos de interés particular de los nietos. Ella es la abuela a la que prefieren visitar con mayor frecuencia (una a dos veces por semana) y en persona; sienten que la conocen y que también es ella quien más los conoce. No obstante, los nietos refirieron que ellas generan poca influencia en sus formas de pensar, lo mismo que los demás abuelos. Los adolescentes sienten que los padres influyen fuertemente en la relación que ellos mantienen con los abuelos, por lo que cabría reflexionar en torno a cómo las madres y los padres de los adolescentes hacen de intermediarios en estas relaciones generacionales.

Existen actividades que forman parte de mandatos sociales y de obligaciones que en general se espera en la relación de los nietos(as) con sus abuelos(as). Es posible

\section{Existen actividades que} forman parte de mandatos sociales y de obligaciones que en general se espera en la relación de los nietos(as) con sus abuelos(as). que las actividades como son las visitas, ver televisión, vacacionar, sean actividades que forman parte de una tradición que involucra mandatos y obligaciones. Sin embargo, algunas de estas actividades se ubican como formas de transmisión del vínculo generacional a través de la cooperación dentro de la selectividad afectiva que mantienen abuelos y nietos. Observamos que existen prescripciones de cómo se debe actuar y comportarse con los nietos de parte de los padres que sin embargo no siempre son obedecidas. La cooperación intergeneracional implica por parte de los nietos la necesidad de elegir por sí mismos y por su voluntad la actividad que van a realizar, así como con cuál de sus abuelos la van a realizar. Al mismo tiempo, parece que tanto para las nietas como para los nietos los intercambios afectivos y comunicacionales no son ilimitados y permanentes, por lo que existen momentos de acercamiento afectivo con espacios de distancia y extrañeza, lo que hace pensar que se está ante procesos permanentes de construcción y reconstrucción de los vínculos generacionales. Existe asimismo una influencia de sexo/género en el sentido de transmisión de determinados modelos de lo que es masculino y femenino. Por el lado de las nietas estas diferencias son aún más significativas.

\section{Bibliografía}

AARP Public Policy Institute. State of 50+ American Survey. Washington, DC: AARP, 2005.

ADAMS, B. N. Cross-cultural and U.S. kinship. In: M. B. Sussman, S. K. Steinmetz, \& G. W. Peterson (Eds.), Handbook of Marriage and the Family. New York: Plenum Press, 1999, pp. 77-92.

BENGSTON, V., ROSENTHAL, C., BURTON, L. Families and Aging: Diversity and Heterogeneity. In: R. H. Binstock \& L. K. George (Eds.), Handbook of Aging and the Social Sciences, San Diego, CA: Academic Press, 1990, pp. 263-287.

BENGTSON, V. L. Beyond the nuclear family: The increasing importance of multigenerational relationships en American society. Journal of Marriage and the Family, 63, 2001, pp. 1-16.

COLEMAN, J. Social capital and the creation of human capital. American Journal of Sociology, 94, 1988, pp. 95-121.

CONAPO, Proyecciones de la Población en México 2010-2050. México, 2013. Disponible en: http://www.conapo.gob.mx/en/CONAPO/ Proyecciones. Consultado el: 20 de jul. 2014.

EHRLE, G.; DAY, H. D. Adjustment and family functioning of grandmothers rearing their grandchildren. Contemporary Family Therapy, 16 (1), 1994, pp. 67-82.

FURSTENBERG, F. F.; HUGHES, M. E. Social capital and successful development among at risk youth. Journal of Marriage and the Family, 57, 1995, pp. 580-592. 
GANONG, L. H.; COLEMAN, M. Stepfamily relationships: Development, dynamics, and interventions. New York: Kluwer Academic/ Plenum Publishers. 2004.

HARPER, S. Changing families as european societies. European Journal of Sociology, 44, Issue 02. 2003, pp. 155-184.

HERNANDEZ, L; RODRIGUEZ, C. Vinculo Generacional entre abuelos y nietos adolescentes: Entre la cooperación y la selectividad. (Tesis inédita de Maestría). Circulo de estudios de Psicología Profunda, México. 2015.

INEGI. Encuesta Nacional sobre la Dinámica de las Relaciones en los Hogares,

Marco conceptual. 2011. Disponible en: http://www.inegi.org.mx/est/contenidos/Proyectos/Encuestas/Hogares/especiales/endireh/ endireh2011/default.aspx. Consultado el 3 de abril de 2012.

KLEIN, A. Nuevas formas de relacionamiento abuelos-nieto adolescentes desdelos cambio demográficos-sociales actuales. Psicología Revista, 18 (1). 2009, pp. 1-25.

Nuevas formas de familias, paternidades y relaciones familiares como modelo de intersecciones intergeneracionales. Ageing Horizons, 9. 2010, pp. 73-81.

. Subjetividad, Familias y Lazo social. Procesos psicosociales emergentes Buenos Aires, Ediciones Manantial, 2013.

LEVER,K.; WILSON, J. Encoreparenting: Whengrandparentsfilltheroleof primary caregiver. The Family Journal: Counseling and Therapy for Couples and families, 13(2), 2005, pp.167-171.

MORAGAS, R. Gerontologia social: envelhecimento e qualidade de vida. São Paulo: Paulinas, 1997.

RIZZINI, I. Crianças, adolescentes e suas bases familiares: tendências e preocupações Globais. In: SOUSA, S. M.; RIZZENI, I. (Eds.). Desenhos de família. Criando os filhos: a família goianiense e os elos parentais. Goiânia: Cânone Editorial, 2001, p. 124-167.

UHLENBERG, P. Historical forces shaping grandparent-grandchild relationships: Demography and beyond. En: SILVERSTEIN, M. (Ed.). Annual review of gerontology and geriatrics. Focus on intergenerational relations across time and place. New York: Springer Publishing Company, Inc., 2005, pp. 77-97.

U. S CENSUS BUREAU. Grandparents living with own Grandchildren under18yearsandresponsibilityfor own grandchildren: Table PCT015of the Census 2001 Supplementary Survey. 2002. Recuperado de: http://factfender.census.gov/servlet/BasicFactsServlet. Consultado el 10 de julio de 2013.

VILLAGÓMEZ ORNELAS, P. El envejecimiento demográfico en México: niveles, tendencias y reflexiones en torno a la población de adultos mayores México, Instituto de Geriatría, 2009.

WAINERMAN, C. Vivir en Familia. Buenos Aires: UNICEF/Losada, 1996.

WIDMER, E. D. Couples and their networks. In: RICHARDS, M.; SCOTT, J.; TREAS, J. (Eds.), Blackwell companion to the sociology of families. London: Blackwell, 2004, pp. 356-373.

WILCOXON, S. A. Grandparents and grandchildren: an often neglected relationship between significant others. Journey of Counseling and Development, 65, 1987, pp. 289-290.

\section{Alejandro León Klein}

alejandroklein@ hotmail.com

Doctor en Trabajo Social por el Universidad Federal de Rio de Janeiro

Profesor Investigador Universidad de Guanajuato, México

\section{Lorena Isis Hernández Basilio}

isishbc@hotmail.com

Master en Psicología Clínica por el Círculo de Estudios de Psicología Profunda, Universidad Nacional Autónoma de México

\section{María Cristina Rodríguez García}

macrisroga@yahoo.com.mx

Master en Psicología Clínica por el Círculo de estudios de Psicología Profunda Universidad Nacional Autónoma de México

Universidad de Guanajuato

Blvd. Puente Milenio \#1001, Predio San Carlos, 37670

León - Guanajuato - México 